\title{
High Toughness Carbon Cloth Composites for Low Temperature Applications
}

\author{
Sara Ronca ${ }^{\mathrm{a}}$, Giuseppe Forte ${ }^{\mathrm{a}}$, Leno Mascia ${ }^{\mathrm{a}}$, Sanjay Rastogi ${ }^{\mathrm{a}}$ \\ ${ }^{a}$ Department of Materials, Loughborough University, Loughborough, LE11 3TU, UK
}

\begin{abstract}
Carbon Fibre Reinforced Polymers based on a thermoplastic, high performance matrix such as Ultra High Molecular Weight Polyethylene have been produced using two different routes and it was found that in-situ polymerization of the matrix is a possible way forward to achieve a combination of high strength and high toughness in composites.
\end{abstract}

Keywords: Carbon Fibre Reinforced Polymers, UHMWPE.

PACS 81

\section{INTRODUCTION}

Carbon Fibre Reinforced Polymers (CFRP) are composite materials made of a carbon fibre component, providing strength and rigidity, and a polymer component to bind the reinforcement. Thermoset matrices have been largely used for this purpose, and they currently find application in a variety of fields where strength and rigidity need to be coupled with lightweight and ease of fabrication. The decreasing cost associated with the manufacturing of CFRP has expanded its use in many fields, which are not limited to aerospace and automotive, but includes civil engineering and sports goods.

The main issue associated with thermosetting matrices is their low resistance to impact. For this reason, much of the research in this area has been directed towards high-performance thermoplastic matrices, such as polyether ether ketone (PEEK), polyetherimide (PEI) and polyethersulfone (PES), which exhibit higher toughness and provides a higher resistance to interlaminar crack growth ${ }^{1}$. The glass transition temperature $\left(T_{\mathrm{g}}\right)$ of these aromatic polymers is well above $100^{\circ} \mathrm{C}$, and, therefore, the matrix will be in its glassy state for a large number of applications, such as civil engineering and sports goods, where normal usage temperatures are below $40-50^{\circ} \mathrm{C}$. To further improve the low temperature toughness, a high-performance engineering thermoplastic with a high ductility would be highly desirable.

The best candidate to fulfil the ductility requirements is Ultra High Molecular Weight Polyethylene (UHMWPE), which has a molecular weight in excess of 2 million $\mathrm{g} / \mathrm{mol}$. Because of its unrivalled ductility and toughness UHMWPE is used as an engineering material rather than a typical thermoplastic, which is complemented by a low coefficient of friction, self-lubrication, high resistance to abrasion, low moisture absorption and high resistance to most chemicals. The main problem associated with this material is the difficulty in processing it in the melt state by conventional processing techniques. When very high molecular weights are reached $\left(M_{\mathrm{w}}>1 * 10^{6} \mathrm{~g} / \mathrm{mol}\right)$, the melt viscosity of the material exceeds the capability limits of these processes, as can be deduced directly by the power law, $\eta_{0}=\alpha M_{\mathrm{w}}^{3.4}$. The high viscosity is related to the molar mass ${ }^{2}$ by the large number of physical entanglements (contact points between the neighbouring chains), which increase with molar mass.

We have recently reported a synthetic method that makes it possible to obtain grades of UHMWPE with improved processability ${ }^{3,4}$, thanks to the reduction of entanglements in the nascent state of the material. At the same time it has been possible to carry out a direct synthesis of UHMWPE composites with a variety of fillers ${ }^{5}$. The obtained 'disentangled' UHMWPE shows also better mechanical properties, while retaining the desired characteristics of light-weight (density is $0.93-0.96 \mathrm{~g} / \mathrm{cm}^{3}$ ) and chemical inertness.

In the present work, we report our findings on the use of this matrix in combination with a carbon fibre cloth to produce high toughness, light-weight composites that are not only potentially cheaper than those produced with 
other high-performance thermoplastic, but can also find application in fields where these characteristics have to be retained even at low temperatures.

Three types of composites were produced and examined. In the first and second type, alternate layers of UHMWPE (commercial or disentangled) and carbon cloth were pressed together, while in the third type the UHMWPE has been synthesised directly on the carbon cloth and the resulting 'pre-impregnated' cloths were processed under the same pressure-heat conditions.

\section{Materials and methods}

\subsection{Materials}

All reactions involving air- and moisture-sensitive compounds were carried out under a dry nitrogen or argon atmosphere using standard Schlenk techniques and a glove-box. The disentangled UHMWPE used in the first set of composites (section 2.2) was synthesised according to reference 4 . The main molecular characteristics were $M_{\mathrm{w}}=$ $5.5 \times 10^{6} \mathrm{~g} / \mathrm{mol}$, Molecular Weight Distribution $(\mathrm{MWD})=2.5$, melting temperature, $T_{\mathrm{m}}=140^{\circ} \mathrm{C}$. For comparison, a commercial UHMWPE was also used, with $M_{\mathrm{w}}=3.7 \times 10^{6} \mathrm{~g} / \mathrm{mol}$, and MWD $=8.4$. The $3 \mathrm{~K}$ plain weave carbon cloth (CC) from Easy Composites Ltd had a density of $1.76 \mathrm{~g} / \mathrm{cm}^{3}$, a tensile strength of $3.53 \mathrm{GPa}$ and a modulus of $230 \mathrm{GPa}$. Prior to use, it was washed with toluene and then dried in a vacuum oven at $40^{\circ} \mathrm{C}$ overnight to remove possible surface contaminants that could interfere with the composite formation or the polymerisation reaction.

\subsection{UHMWPE - Carbon Cloth Composites}

The first and second set of composites was produced using a combination of heat and pressure in a hydraulic press, according to the following procedure. In a steel mould having a cavity of lateral dimensions $10 \times 10 \mathrm{~cm}$ and variable thickness, a layer of carbon cloth was placed between two evenly-spread layers of UHMWPE powder. The mould was closed and subject to increasing forces (from 1 tonne to 20 tonne) over a period of 25 minutes in a hydraulic press heated at $125^{\circ} \mathrm{C}$. Owing to the improved processability of the disentangled UHMWPE, the polymer matrix was perfectly compact even when processed below the melting temperature. For the commercial UHMWPE this step had to be completed at $160^{\circ} \mathrm{C}$. Afterwards, 4 layers of sintered UHMWPE/carbon cloth composite were stacked in the mould and compressed with increasing forces (from 1 tonne to 20 tonne) over a period of 25 minutes at $190^{\circ} \mathrm{C}$. The relative amount of cloth and UHMWPE was adjusted in order to give a weight ratio of 50/50 in the final product. Specimens of suitable dimension for mechanical and thermal tests were cut with a band saw.

\section{3 'Pre-impregnated' Carbon Cloth Composites via In-situ Polymerisation}

The third set of composites was realised by in-situ synthesis of UHMWPE on the carbon cloth, following the procedure described in reference 5, with minor modifications. In the glove-box, the carbon cloth was sprayed with a toluene solution of pre-catalyst/MAO, then placed in a cylindrical steel vessel. The vessel was closed under argon atmosphere, brought out from the glove-box, then connected to the ethylene gas-line and pressurised at the desired value. The ethylene consumption, due to the ongoing polymerisation, was monitored with an on-line mass flowmeter, and the vessel was de-pressurised (thus quenching the polymerisation reaction) when the desired amount of ethylene had been consumed. The resulting 'pre-impregnated' cloth was washed with acetone and dried in a vacuum oven at $40^{\circ} \mathrm{C}$ overnight. The cloth was cut in pieces of suitable dimension; the pieces were layered in the steel mould and subject to the same pressure/heat treatment ( 1 to 20 tonnes, $190^{\circ} \mathrm{C}$ ) of the previous set.

\subsection{Characterisation of Composites}

The interfacial adhesion was investigated by the cross sections of the carbon cloth composites using a LEO 1539 VP Gemini field emission scanning electron microscope (FEGSEM). All the samples for microscopy were cut $45^{\circ}$ along the fibres.

The three-point bending tests were carried out on a Lloyd LR50K Plus machine with a load cell of 1000N. The span to thickness ratio was controlled at 20 for all the composites. The specimens were tested at the speed of 10 $\mathrm{mm} / \mathrm{min}$ and were stopped when the load dropped to $80 \%$ of the maximum load that was recorded. Two specimens from each composite were tested when the two results were close to each other, otherwise a repeated trial was necessary.

\section{Results and Discussions}

Following the method described in the Experimental section, three samples of UHMWPE/Carbon cloth composites were prepared (Table 1) and the void content calculated according to the equation (1), where $W, L$ and $D$ are the sample width, length and thickness, and $M$ and $d$ represent mass and density: 


$$
. \text { Void } \%=\frac{W \times L \times D_{\text {average }}-M_{\text {polymer }} / d_{\text {polymer }}-M_{\text {cloth }} / d_{\text {cloth }}}{W \times L \times D_{\text {average }}} \times 100
$$

TABLE 1. Void content of the three composites samples

\begin{tabular}{lcc}
\hline Sample & Characteristics of UHMWPE & Void content \\
\hline A & Disentangled UHMWPE & $15.8 \%$ \\
B & Commercial UHMWPE & $16.9 \%$ \\
C & In-situ polymerized UHMWPE & $0.6 \%$ \\
\hline
\end{tabular}

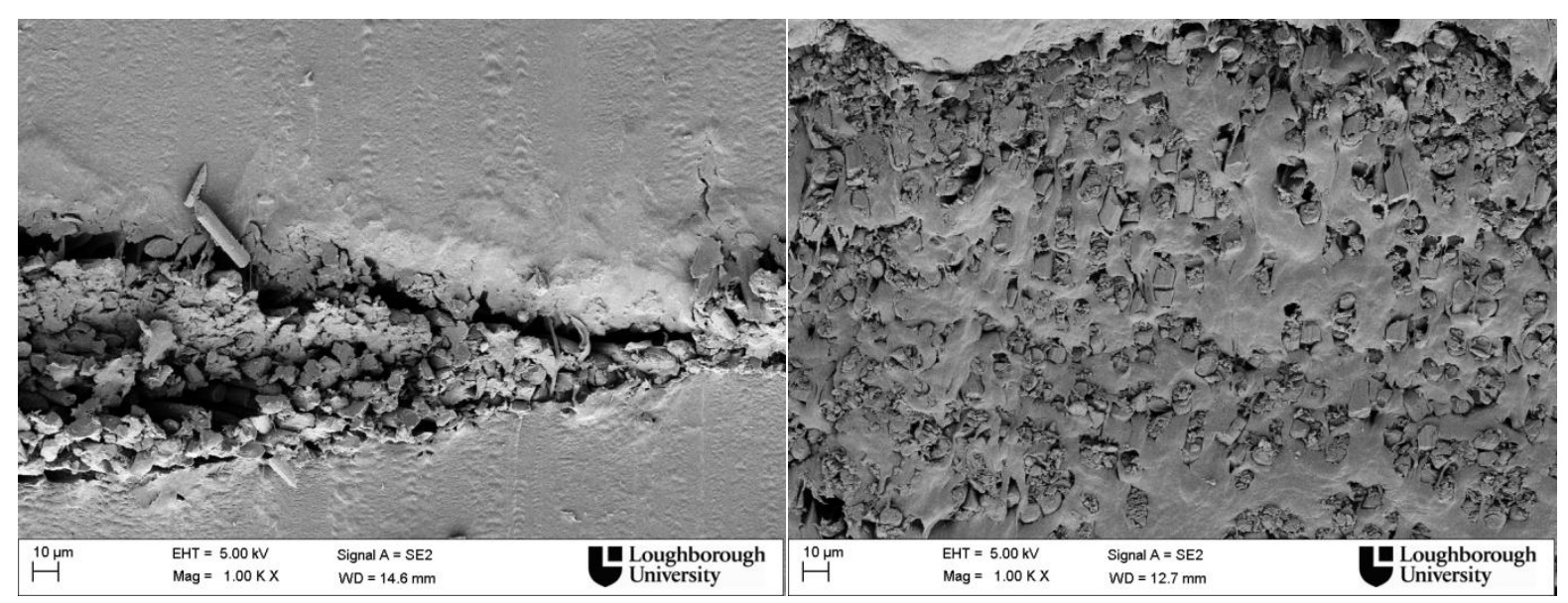

Figure 1. SEM images of the cross section of sample A (left) and C (right), showing the better interpenetration of the thermoplastic matrix for the in-situ sample.

The better processing characteristics of disentangled UHMWPE are confirmed by the fact that well sintered layers of UHMWPE-CC can be obtained below the melting $\mathrm{T}$ of the polymer $\left(125^{\circ} \mathrm{C}\right)$, while for the commercial material this was impossible, and a higher temperature had to be used $\left(160^{\circ} \mathrm{C}\right)$. However, when subject to the stacking process at $190^{\circ} \mathrm{C}$ the two samples behave very similarly, as it should be expected from the similar molecular weights of the two polymers. Possibly, the slightly lower molecular weight and larger MWD of the commercial sample improves polymer flowability at higher temperature, thus reducing the observable differences between the two samples, as we have shown elsewhere ${ }^{6}$. In the case of sample $C$, the situation is dramatically improved by the fact that the UHMWPE is grown around the individual fibres of the cloth, as shown in Figure 1 and 2.
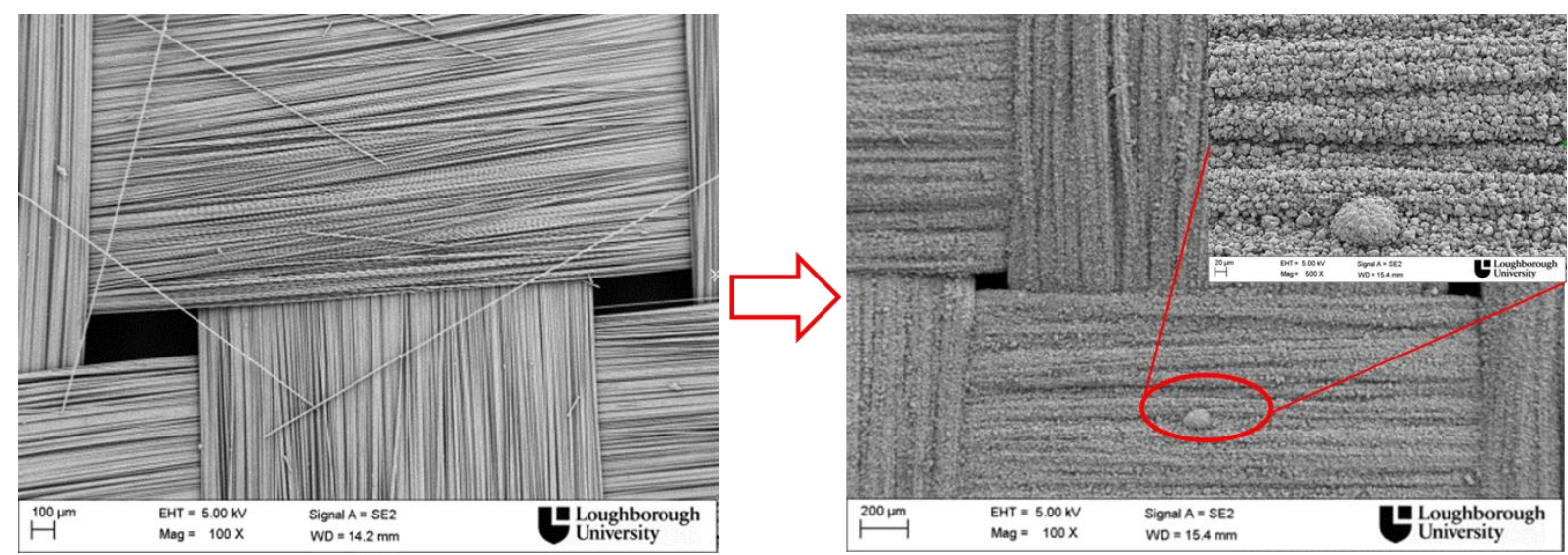

Figure 2. SEM images of plain CC(left) and after in-situ polymerization of UHMWPE (right), showing the even coverage of the polymer matrix on the individual fibres. 

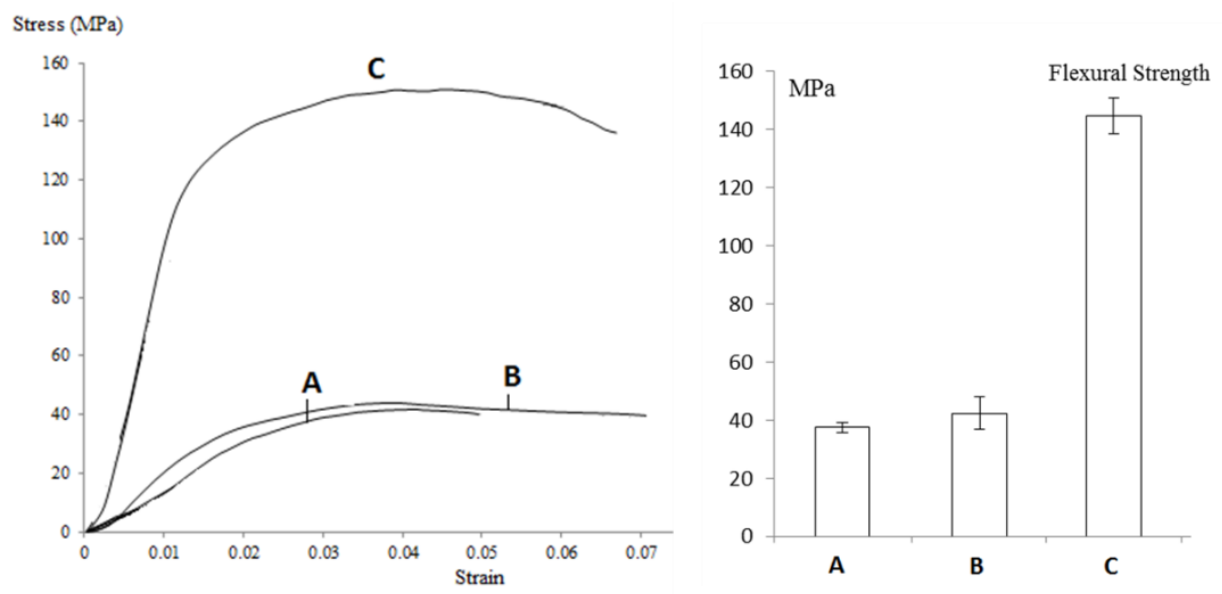

Figure 3. Stress-strain curves as obtained from the three-point bending tests (left) and corresponding flexural strength of the samples (right).

The results of the three-point bending test show that samples A and B have very similar performances, as it should be expected from the very similar molecular weights of the UHMWPE matrix, while sample C outperform both with a flexural strength that is more than tripled. It is interesting to note that the failure mechanism for $\mathrm{C}$ is quite different than A and B (Figure 4): while the latter fail by a buckling-driven delamination, where the most superficial layer separates from the layers below, the former fails by formation of a kink band, usually associated with high interfacial adhesion.
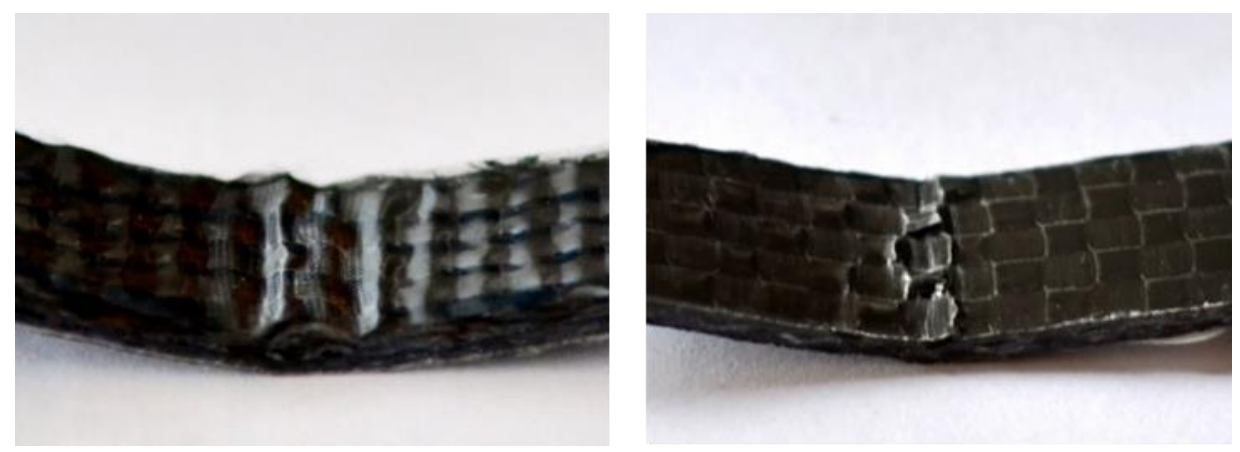

Figure 4. Failure at the mid-span of sample A (left) and C (right).

\section{Conclusions}

The possibility to produce high-performance composites using a highly ductile thermoplastic matrix (UHMWPE) in combination with carbon cloth has been investigated. The poor adhesion between the cloth and the aliphatic matrix is counteracted by the use of an in-situ polymerization method that allows for an even and deep coverage of the individual fibres with UHMWPE, achieving a flexural strength of $150 \mathrm{MPa}$.

\section{REFERENCES}

1] Christopoulos, G.C.; Paipetis, S.A.; Walter, R.; Friedrich, K. J. Thermoplastic Comp. Mat. 1995, 8, 193-207.

2] De Gennes, P.G. J. Chem. Phys. 1971, 55, 572-579.

3] Rastogi, S.; Lippits, D.R.; Peters, G.W.M.; Graf, R.; Yao, Y.; Spiess, H.W. Nature Materials 2005, 4, 635-641

4] Rastogi S.; Yao Y.; Ronca S.; Bos J.; van der Eem, J. Macromolecules 2011, 44, 5558-5568

5] Ronca, S.; Forte, G.; Tjaden, H.; Yao, Y.; Rastogi, S. Polymer 2012, 53, 2897-2907.

6] Liu, K.; Ronca, S.; Andablo-Reyes, E.; Forte, G.; Rastogi, S. Macromolecules 2015, 48, 131-139. 